\title{
Prenatal exome sequencing in anomalous fetuses: new opportunities and challenges
}

\author{
Neeta L. Vora, MD ${ }^{1}$, Bradford Powell, MD, PhD², Alicia Brandt, MS², Natasha Strande, PhD ${ }^{3,4}$, \\ Emily Hardisty, MS, CGC ${ }^{1}$, Kelly Gilmore, MS, $\mathrm{CGC}^{1}$, Ann Katherine M. Foreman, MS, $\mathrm{CGC}^{2,5}$, \\ Kirk Wilhelmsen, $\mathrm{MD}, \mathrm{PhD}^{6}$, Chris Bizon, $\mathrm{PhD}^{6}$, Jason Reilly, $\mathrm{BA}^{6}$, Phil Owen, $\mathrm{BS}^{6}$, \\ Cynthia M. Powell, MD, MS ${ }^{2,7}$, Debra Skinner, PhD, MA ${ }^{8}$, Christine Rini, PhD ${ }^{9}$, \\ Anne D. Lyerly, MD, MA ${ }^{10}$, Kim A. Boggess, MD ${ }^{1}$, Karen Weck, MD ${ }^{3,4}$, \\ Jonathan S. Berg, MD, $\mathrm{PhD}^{2}$ and James P. Evans, MD, PhD ${ }^{2,10}$
}

\begin{abstract}
Purpose: We investigated the diagnostic and clinical performance of exome sequencing in fetuses with sonographic abnormalities with normal karyotype and microarray and, in some cases, normal gene-specific sequencing.

Methods: Exome sequencing was performed on DNA from 15 anomalous fetuses and from the peripheral blood of their parents. Parents provided consent to be informed of diagnostic results in the fetus, medically actionable findings in the parents, and their identification as carrier couples for significant autosomal recessive conditions. We assessed the perceptions and understanding of exome sequencing using mixed methods in 15 mother - father dyads.
\end{abstract}

Results: In seven (47\%) of 15 fetuses, exome sequencing provided a diagnosis or possible diagnosis with identification of variants in the following genes: COL1A1, MUSK, KCTD1, RTTN, TMEM67, PIEZO1 and DYNC2H1. One additional case revealed a de novo nonsense mutation in a novel candidate gene (MAP4K4). The perceived likelihood that exome sequencing would explain the results (5.2 on a 10-point scale) was higher than the approximately $30 \%$ diagnostic yield discussed in pretest counseling. Conclusion: Exome sequencing had diagnostic utility in a highly select population of fetuses where a genetic diagnosis was highly suspected. Challenges related to genetics literacy and variant interpretation must be addressed by highly tailored pre- and posttest genetic counseling.

Genet Med advance online publication 18 May 2017

Key Words: counseling; diagnosis; ethics; exome; prenatal

\section{INTRODUCTION}

Congenital anomalies affect 2 to $4 \%$ of all infants and are responsible for $20 \%$ of perinatal deaths. ${ }^{1}$ Currently, prenatal diagnosis begins with a positive serum or cell-free DNA screen for aneuploidy. This is followed by a targeted anatomical survey and diagnostic tests, such as chorionic villus sampling or amniocentesis. Standard karyotypes and microarrays are obtained from chorionic villi or amniocytes, or, if specific pathogenic variants are known in the parents, targeted sequencing is performed. While microarrays increase the diagnostic yield above that of standard karyotypes alone, 80 to $90 \%$ of anomalous fetuses with a normal karyotype also have a normal microarray and thus remain without a definitive diagnosis. ${ }^{2,3}$ Additional molecular genetic testing, either on single genes or panels driven by phenotype, may be performed if indicated and if a limited differential diagnosis suggests that such targeted sequencing will be beneficial. Exome sequencing, which provides sequence data from the exons (coding regions) of known genes in the human genome, has proven to be a powerful diagnostic tool in adults and children with genetic disorders, such as birth defects and intellectual disability. 4,5 Compared with a $10 \%$ diagnostic rate using karyotyping with microarray, exome sequencing demonstrated diagnostic rates of approximately $30 \%$ in a postnatal cohort of patients with birth defects. ${ }^{4}$ The use of exome sequencing of fetal DNA obtained by amniocentesis has been reported in isolated cases. ${ }^{6,7}$ Small case series reporting the increased diagnostic utility of exome sequencing prenatally after a normal microarray have also been published showing diagnostic rates ranging from 10 to $57 \% .^{8-10}$ Thus,

\footnotetext{
${ }^{1}$ Division of Maternal-Fetal Medicine, Department of Obstetrics and Gynecology, University of North Carolina at Chapel Hill, Chapel Hill, North Carolina, USA; ${ }^{2}$ Department of Genetics, School of Medicine, University of North Carolina at Chapel Hill, Chapel Hill, North Carolina, USA; ${ }^{3}$ Department of Pathology and Laboratory Medicine, University of North Carolina at Chapel Hill, Chapel Hill, North Carolina, USA; ${ }^{4}$ Renaissance Computing Institute, University of North Carolina at Chapel Hill, Chapel Hill, North Carolina, USA; ${ }^{5}$ North Carolina Translational and Clinical Sciences Institute, University of North Carolina at Chapel Hill, Chapel Hill, North Carolina, USA; ${ }^{6}$ Department of Genetics and Department of Neurology, Renaissance Computing Institute, University of North Carolina at Chapel Hill, Chapel Hill, North Carolina, USA; ${ }^{7}$ Department of Pediatrics, Division of Genetics and Metabolism, University of North Carolina at Chapel Hill, Chapel Hill, North Carolina, USA; ${ }^{8}$ Frank Porter Graham Child Development Institute, University of North Carolina at Chapel Hill, Chapel Hill, North Carolina, USA; ${ }^{9}$ Department of Health Behavior, University of North Carolina at Chapel Hill, Chapel Hill, North Carolina, USA; ${ }^{10}$ Department of Social Medicine and Center for Bioethics, University of North Carolina at Chapel Hill, Chapel Hill, North Carolina, USA. Correspondence: Neeta L. Vora (neeta_vora@med.unc.edu)
} 
exome sequencing appears to be a promising technique to fill the existing diagnostic gap for fetal diagnosis.

Exome sequencing appears to be a promising technique because it has increased diagnostic capability when karyotyping and microarrays are normal and because it is less costly and more clinically applicable than whole-genome sequencing. Our aim was to examine the utility of exome sequencing for prenatal diagnosis in noncontinuing (defined as pregnancy termination, intrauterine fetal demise, or neonatal death in the delivery room) pregnancies with multiple anomalies and normal results with standard prenatal genetic diagnostic tests (karyotyping and microarray). Targeting this population for initial study focuses on those families with the greatest need while avoiding some of the ethical complexities of communicating risk or study findings to those with ongoing pregnancies. Additionally, because of the unique challenges related to the implementation of exome sequencing prenatally, we sought to understand maternal perspectives, expectations, and understanding of fetal genetic results obtained by exome sequencing.

\section{MATERIALS AND METHODS}

Mother - father - fetus trios in pregnancies complicated by a fetus with multiple congenital anomalies were identified from the University of North Carolina (UNC) at Chapel Hill prenatal diagnosis clinics (Chapel Hill and Raleigh, North Carolina) between July 2014 and July 2016. Approval from the UNC at Chapel Hill Institutional Review Board (13-4084) was obtained before patient consent and enrollment. Inclusion criteria included: (i) a pattern of anomalies highly suggestive of an underlying genetic disorder; (ii) an unknown diagnosis based on karyotyping, microarray, and, in some cases, gene-specific sequencing; and (iii) the availability of fetal and parental DNA. Trios were identified prospectively and retrospectively, enabling us to obtain fetal specimens at various gestational ages. Prospectively, women pregnant with a singleton fetus suspected to have a lethal anomaly consistent with a genetic disorder were approached for participation after they had made the decision to continue the pregnancy. In the case of noncontinuing pregnancies, the study was not mentioned or offered until the couple had made a decision to terminate the pregnancy. Retrospective identification of potential trios was accomplished by querying the UNC Perinatal Database to identify women with a history of fetal or neonatal death who had not received an explanatory diagnosis by standard prenatal testing. We contacted women for potential enrolment if they had previously indicated a desire to be re-contacted should additional fetal testing options become available and if they had fetal cells archived and available for DNA extraction. Additional participants in the retrospective cohort were either self-referred or referred by a clinician aware of our current study recruitment. Once participants were enrolled, we collected parental blood and retrieved stored fetal samples for exome sequencing analysis. After the first seven trios were enrolled, we expanded enrolment to individuals not receiving care at UNC; Skype was used to facilitate counseling, consent, and the discussion of results in nonlocal cases. The sample size of 15 trios is a convenience sample for this pilot study.

Mothers and fathers from both retrospective and prospective groups had pretest counseling about exome sequencing and the possible results it can provide. Consent was obtained separately from mothers and fathers; both were informed about the possibility of exome sequencing revealing nonpaternity. Participants were given the option to opt out at any time during the study. Owing to the complexity of the genetic information that results from exome sequencing, consent and the return of results were performed by a certified genetic counselor who was not involved in the patient's clinical care. This was to avoid bias and undue pressure on the patient to participate. All participants agreed to be informed of: (i) any diagnostic findings with the potential to explain the fetal phenotype; (ii) any incidental findings in a parent that would have medically actionable implications for that parent; ${ }^{11}$ and (iii) carrier status for significant autosomal recessive conditions for which both parents were carriers. Diagnostic results were classified into seven categories (Table 1). More than one result could be provided for a trio. After consent, we obtained parental blood and extracted DNA in the Biospecimen Processing Facility (BSP) or, for nonlocal cases, received DNA directly from an outside institution. If previously isolated DNA was not available, we extracted fetal DNA from stored products of conception, fetal amniocytes, or villi (retrospective) or from umbilical cord blood, amniocytes, or chorionic villi (prospective). We split the DNA and sent a duplicate sample to the UNC Molecular Genetics Laboratory, a Clinical Laboratory Improvement Amendments (CLIA)certified and College of American Pathologists-accredited facility, where it was stored and used for Sanger sequencing confirmation of genetic variants. Duplicate samples streamline the process of variant confirmation and allow for quality checks between samples, as well as ensuring their eligibility for inclusion in the medical record. After confirmation using Sanger sequencing, parents were given the option to sign a separate consent form to have their own or their deceased child's variants placed in the electronic medical record.

\section{Exome sequencing and variant analysis}

We created exome sequencing libraries and exome capture from maternal, paternal, and fetal DNA samples, as previously described, ${ }^{12}$ and transferred them to the UNC High-Throughput Sequencing Facility for sequencing using the Illumina Hi-Seq 2500. We processed, mapped, and aligned raw-read data, and identified variants using a standard bioinformatics pipeline developed for the next-generation exome sequencing (NCGENES) project in collaboration with colleagues in the Department of Genetics and the Renaissance Computing Institute. ${ }^{13}$

We captured quality metrics at all stages of processing to determine whether outputs could be used for analysis. Metrics included checks on input file correctness, distributions of 
Table 1 Classification scheme of case-level results

\begin{tabular}{|c|c|}
\hline Classification & Description \\
\hline \multicolumn{2}{|l|}{ Positive } \\
\hline Positive-definitive & $\begin{array}{l}\text { Known pathogenic variant(s) in a known disease gene and consistent with the inheritance pattern; fetal phenotype } \\
\text { consistent with the reported disease spectrum. }\end{array}$ \\
\hline Positive-probable & $\begin{array}{l}\text { Likely pathogenic variant(s) in a known disease gene and consistent with the inheritance pattern; fetal phenotype consistent } \\
\text { with the reported disease spectrum. }\end{array}$ \\
\hline Positive-possible & $\begin{array}{l}\text { A single rare or novel VUS known to be in trans with a pathogenic/known pathogenic variant in a gene that explains the } \\
\text { phenotype. }\end{array}$ \\
\hline \multicolumn{2}{|l|}{ Uncertain } \\
\hline Uncertain-VUS & $\begin{array}{l}\text { VUS in a known disease gene and consistent with the inheritance pattern; fetal phenotype consistent with the reported } \\
\text { disease spectrum (e.g., uncertainty is limited to the pathogenicity of the variant). }\end{array}$ \\
\hline Uncertain-AR Het & $\begin{array}{l}\text { Single heterozygous variant (known pathogenic, likely pathogenic, or highly suspicious VUS) identified in a disease gene } \\
\text { implicated in a recessive condition; fetal phenotype consistent with the reported disease spectrum. }\end{array}$ \\
\hline Uncertain-contributory & $\begin{array}{l}\text { Known pathogenic or likely pathogenic variant(s) in a known disease gene, but fetal phenotype is not completely } \\
\text { consistent with the reported disease spectrum and thus the finding may contribute to, but not completely explain, the } \\
\text { phenotype. }\end{array}$ \\
\hline Uncertain-other & $\begin{array}{l}\text { Findings of uncertain case-level significance, including potential novel gene discoveries. For example, predicted deleterious } \\
\text { variant(s) in a novel candidate gene that has not previously been implicated in human disease or for which the published } \\
\text { data to support human disease association may not yet be definitive. Supporting data could be based on model organism } \\
\text { data, copy-number variation data, the tolerance of the gene to sequence variation, data about tissue or developmental } \\
\text { timing of expression, or knowledge of the gene function and pathway analysis. Further research is required to evaluate any } \\
\text { of the suggested candidate genes. }\end{array}$ \\
\hline \multicolumn{2}{|l|}{ Negative } \\
\hline Negative & No variants in genes associated with the reported phenotype identified. \\
\hline
\end{tabular}

AR Het, autosomal recessive-heterozygous; VUS, variant of uncertain significance.

Compiled from refs. 10,18,20, and 21.

Table 2 Demographics of the mothers

\begin{tabular}{lc} 
Characteristics & Study cohort $(\boldsymbol{n}=\mathbf{1 5})$ (count (\%)) \\
\hline Age (years) & $32.0 \pm 5.11(22-39)$ \\
\hline Mean \pm s.d. (range) & $14(93.3)$ \\
Race & $1(6.6)$ \\
\hline Caucasian (white) & $1(6.6)$ \\
\hline African American & $11(73.3)$ \\
\hline Education level & $3(20.0)$ \\
\hline High school graduate or equivalent & \\
\hline College education & $4(26.6)$ \\
\hline Graduate or professional degree & $5(33.3)$ \\
\hline Total family income & $6(40.0)$ \\
\hline 444,999 or less & $8(53.3)$ \\
$\$ 45,000-89,999$ & $7(46.6)$ \\
$\$ 90,000$ or higher & \\
\hline Previous genetic testing to look for causes of health problems \\
\hline Yes & $13(86.7)$ \\
\hline No & $2(13.3)$ \\
\hline Married & \\
\hline Yes & \\
\hline No & \\
\hline
\end{tabular}

nucleotide and quality scores, percent of reads aligned, read gap distributions, percent of reads with pairs, coverage across the genome and from targeted regions, and variants identified by the genome analysis toolkit (GATK). Variants were annotated with information regarding predicted molecular effect (SnpEff $)^{14}$ and population allele frequencies (Exome Aggregation Consortium). ${ }^{15}$ These additional annotations and trio data were used to filter and prioritize variants according to inheritance patterns (de novo, compound heterozygous variants, and homozygous recessive variants) within the trio using GEMINI. ${ }^{16}$ Similar to whole-exome sequencing used postnatally, all protein coding regions of the genome were interrogated. We also used a "gene list prioritization" approach to present all known pathogenic, rare truncating, and rare missense variants in genes known to have an association with the fetal phenotype (examples of such gene lists are shown in the Supplementary Tables online). Gene lists specific to the phenotype in question were curated using the primary literature and by reviewing previously developed panels currently in use. When no finding was identified using a gene list, all homozygous variants and compound heterozygous variants in autosomal recessive disorders, and de novo variants in autosomal dominant disorders were manually reviewed. Variants were manually reviewed for potential function in relation to the phenotype by molecular analysts using multiple sources (e.g., mutation databases, the Online Mendelian Inheritance in Man database, PubMed, and the Exome Aggregation Consortium).

A committee of clinical and laboratory geneticists, obstetricians, genetic counselors, and pediatricians who were not 
involved in the patient's clinical care reviewed all the findings of the molecular analysts to make a final determination about return to participants and result classification (e.g., positiveprobable, uncertain-VUS, etc.) using criteria developed by Richards et al. (Table 1) ${ }^{17}$ All variants thought to be potentially causative were reviewed by the committee within 2 weeks of the primary analyst identifying the variant. Results believed to clearly (or possibly) explain the fetal phenotype were reported to parents after confirmation in a CLIA-certified molecular genetics clinical laboratory. In addition, all parental samples were analyzed for a small subset of "medically actionable" genes (e.g., BRCA1/2) per the American College of Medical Genetics and Genomics standards and guidelines, and any findings in the parents were reported. ${ }^{11,17-21}$ Parents also consented to the return of carrier status for significant autosomal recessive conditions for which both parents are carriers. All reported variants, whether diagnostic or incidental, were confirmed by Sanger sequencing in a CLIA-certified molecular genetics laboratory. The diagnostic results were categorized into eight categories (Table 1). ${ }^{11,21,22}$

\section{Assessment of maternal perspectives and understanding}

We completed a mixed methods assessment using questionnaires and semi-structured interviews with 15 mothers. We focused on the mothers' perspectives and understanding in this pilot study. After informed consent, each mother completed a presequencing questionnaire (eight questions related to demographics) and literacy genomic knowledge scale (25 true-or-false questions to assess recall and understanding of the structure and function of genes, how they are inherited, their relation to health, and the strengths and limitations of exome sequencing). The literacy assessments were modified for prenatal use from previously used scales from the NCGENES project. A Wilcoxon rank sum test was performed to compare literacy genomic knowledge scores with income levels, and a $P$-value of less than or equal to 0.05 was defined as significant. This was followed by a semistructured, in-person interview ( $\sim 45 \mathrm{~min})$ with each mother to identify expectations, understanding, and perceptions. To reduce bias, a trained research assistant rather than the genetic counselor or principal investigator (N.L.V.) conducted this interview, which was adapted from a study of diagnostic genome sequencing in adult and pediatric patients conducted as part of the NCGENES project.

A trained research assistant conducted follow-up telephone, quantitative and interview assessments with each mother 4 weeks after the return of results to measure their understanding of the information and its impact on future decisions.

\section{RESULTS}

The participant demographics of the cohort are listed in Table 2. Most (13 of 15) participants enrolled shortly after routine fetal genetic testing (chorionic villus sampling or amniocentesis for karyotyping and microarray) was completed. All enrolled pregnancies had normal results for both karyotyping and single nucleotide polymorphism prenatal microarray. However, 2 of 15 were enrolled 5-10 years after the previous affected pregnancy (cases 1 and 2). The turnaround time to identify pathogenic variants once sequencing was performed ranged from 0 to 28 days (mean: 21 days). Gene lists were developed and used for cases with skeletal findings, nonimmune hydrops, and genitourinary abnormalities. Two of the three skeletal cases were diagnosed using the skeletal dysplasia gene list prioritization approach (COL1A1 and DYNC2H1) and had the shortest turnaround time (0 days to identify pathogenic variants once sequencing data were available).

\section{Molecular diagnoses}

Genotype and phenotype data are listed in Table 3. Further details about the variants can be found in Table 4. In 7 (47\%) of 15 trios, exome sequencing provided a diagnosis or possible diagnosis of the following disorders: osteogenesis imperfecta type 3 (COL1A1); fetal akinesia sequence (MUSK); scalp-ear-nipple syndrome (KCTD1); primordial microcephaly-dwarfism syndrome (RTTN); Meckel-Gruber syndrome (TMEM67); lymphatic dysplasia (PIEZO1); and short rib polydactyly syndrome (DYNC2H1). Of the mutations found, there were two de novo mutations in the proband fetuses (COL1A1 and KCTD1) and five autosomal recessive disorders (MUSK, RTTN, TMEM67, PIEZO1 and DYNC2H1) conferring a $25 \%$ risk of recurrence in a subsequent pregnancy. Exome sequencing provided evidence for expanding the phenotype in one of these syndromes (scalp - ear-nipple syndrome) to the fetal period. There was a significant family history in only one fetal case (case 5: fourth pregnancy affected with arthrogryposis phenotype). Two cases-case 7 (short rib polydactyly) and case 9 (Meckel-Gruber syndrome)-had sufficient ultrasound findings to enable the provider to send the correct gene-specific panel for the specific phenotype of interest. Although other variants in our positive diagnoses could be detected by a gene panel (case 1: osteogenesis imperfecta), the ultrasound phenotype was not detailed enough (shortened long bones with bowing) for the provider to reliably pick the correct panel using the ultrasound findings alone. In addition, autopsy and skeletal survey findings can suggest the wrong diagnosis (for case 1, the autopsy and skeletal survey suggested hypophosphatasia when osteogenesis imperfecta type 3 was the diagnosis). This would have led the provider astray.

Demonstrating the potential of exome sequencing in fetuses to reveal new candidate genes for developmental disorders, in one case with a complex cardiac defect and abnormal kidney location, a de novo stop gain mutation was found in MAP4K4. This gene is known to be integrally involved in vascular development and cell migration and is embryonic lethal in knockout mouse models, but no human phenotype has yet been described. ${ }^{23}$ Since this gene has not been associated with 
Table 3 Phenotype and genotype information for the cohort

Case ID Ultrasound findings Postnatal/autopsy results

\begin{tabular}{|c|c|c|c|c|c|}
\hline & & Postnatal/autopsy results & & & \\
\hline 1 & $\begin{array}{l}\text { Skeletal dysplasia, shortened long } \\
\text { bones, and bowing }\end{array}$ & $\begin{array}{l}\text { Skeletal survey postmortem showed shorted long bones in } \\
\text { the upper and lower extremities, limited ossification of all } \\
\text { bones with multiple fractures noted, and broad metaphyses } \\
\text { of the humeri and tibia; autopsy and skeletal surveys raise } \\
\text { suspicion of hypophosphatasia }\end{array}$ & $\begin{array}{l}\text { De novo splice site mutation in COL1A1 } \\
\text { consistent with osteogenesis imperfecta }\end{array}$ & $\begin{array}{l}\text { c. } 1875+1 \mathrm{G}>\mathrm{A} \\
\text { likely pathogenic }\end{array}$ & $\begin{array}{l}\text { Positive- } \\
\text { probable }\end{array}$ \\
\hline 2 & Postnatal exam showed skin sloughing & Not applicable & $\begin{array}{l}\text { De novo rare missense mutation in the BTB } \\
\text { domain of the KCTD1 gene, which is } \\
\text { associated with scalp - ear - nipple syndrome }\end{array}$ & $\begin{array}{l}\text { c.86A }>\text { G } \\
\text { p.Asn29Ser; } \\
\text { likely pathogenic }\end{array}$ & $\begin{array}{l}\text { Positive- } \\
\text { probable }\end{array}$ \\
\hline 3 & $\begin{array}{l}\text { Severely malformed calvarium, } \\
\text { microcephaly intracranial cyst, growth } \\
\text { restriction, cerebellar hypoplasia, and } \\
\text { absent corpus callosum }\end{array}$ & Not applicable & $\begin{array}{l}\text { Compound heterozygous missense mutations } \\
\text { in the RTTN gene, which cause brain } \\
\text { malformations, microcephaly, growth } \\
\text { retardation, and ciliary dysfunction }\end{array}$ & $\begin{array}{l}\text { c. } 4480 C>T \\
\text { p.Thr1398Met; } \\
\text { VUS }\end{array}$ & Uncertain-VUS \\
\hline
\end{tabular}

absent corpus callosum

$$
\text { c.5143 A>G }
$$

p.Asn1715Asp;

VUS

$\begin{array}{ll}4 \quad \text { Fetal akinesia sequence } & \begin{array}{l}\text { Autopsy consistent with fetal akinesia sequence; no } \\ \text { additional findings }\end{array}\end{array}$

Compound heterozygous missense mutations in the MUSK gene associated with fetal akinesia sequence

$\begin{array}{ll}\text { c. } 1724 \mathrm{~T}>\mathrm{C} & \text { Positive- } \\ \text { p. Ile575Thr; } & \text { possible } \\ \text { likely pathogenic } & \end{array}$

p. Ile575Thr;

possible

\section{c. $2408 \mathrm{~A}>\mathrm{G}$}

p.Tyr803Cys;

VUS

Four affected pregnancies with an Not applicable arthrogryposis phenotype

One rare missense mutation in the CHRND gene, which causes lethal multiple pterygium syndrome; copy-number-variant studies

c.817G >A

p.Asp273Asn;

Uncertain-AR recommended to identify deletion not found

by microarray or exome sequencing

Suspected autosomal recessive

Not applicable

polycystic kidney disease; large

echogenic kidneys; anhydramnios

$5 \quad$ Suspected short rib polydactyly

Postnatal exam by a pediatric geneticist showed severe micromelia, bilateral postaxial hexadactyly, microcephaly, and dysmorphic facial features; autopsy showed gut malrotation hepatomegaly, enlarged kidneys, and abnormal position of heart vessels
One rare missense mutation in the PKHD1

gene; copy-number-variant studies

recommended to identify deletion not found

by microarray or exome sequencing

Compound heterozygous mutations in the

DYNC2H1 gene, which causes short rib

polydactyly
Het

$\begin{array}{ll}\text { c. } 1342 \mathrm{G}>\mathrm{C} & \text { Uncertain-AR } \\ \text { p. Gly448Arg; } & \text { Het }\end{array}$

VUS

c. 10594C > T Positive-

p.Arg3532Ter; possible

novel nonsense

mutation; likely

pathogenic

c. $8012 T>C$

p.Met2671Thr;

missense variant

previously reported in

an affected individual;

considered a VUS 
$\vec{N} \quad$ Table 3 Continued

\begin{tabular}{|c|c|c|c|c|c|}
\hline Case ID & Ultrasound findings & Postnatal/autopsy results & Exome sequencing result & Variant(s) & Classification \\
\hline \multirow[t]{2}{*}{6} & Nonimmune hydrops & Not applicable & $\begin{array}{l}\text { Compound heterozygous mutations in the } \\
\text { PIEZO1 gene, which has been recently } \\
\text { associated with autosomal recessive } \\
\text { generalized lymphatic dysplasia and } \\
\text { nonimmune hydrops fetalis }\end{array}$ & $\begin{array}{l}\text { c. } 307 \mathrm{C}>\mathrm{T} \\
\text { p. Arg103Ter; } \\
\text { novel nonsense } \\
\text { mutation; } \\
\text { likely pathogenic }\end{array}$ & $\begin{array}{l}\text { Positive- } \\
\text { possible }\end{array}$ \\
\hline & & & & $\begin{array}{l}\text { c. } 7129+1 G>C \\
\text { Novel splice site } \\
\text { variant; VUS }\end{array}$ & \\
\hline \multirow[t]{8}{*}{7} & $\begin{array}{l}\text { Multiple brain anomalies including } \\
\text { Dandy Walker malformation, absent } \\
\text { cerebellum, and encephalocele; large } \\
\text { echogenic kidneys, oligohydramnios, } \\
\text { and hands not well visualized }\end{array}$ & Not applicable & $\begin{array}{l}\text { Compound heterozygous mutations in the } \\
\text { TMEM67 gene, which causes Meckel - Gruber } \\
\text { syndrome type } 3 \text { and Joubert syndrome type } 6 \text {; } \\
\text { both previously described; known pathogenic }\end{array}$ & $\begin{array}{l}\text { c.579_580delAG } \\
\text { p.Gly195fs; } \\
\text { previously described; } \\
\text { known pathogenic }\end{array}$ & $\begin{array}{l}\text { Positive- } \\
\text { definitive }\end{array}$ \\
\hline & & & & $\begin{array}{l}\text { c. } 622 \mathrm{~A}>\mathrm{T} \\
\text { p.Arg208Ter; } \\
\text { previously described; } \\
\text { known pathogenic }\end{array}$ & \\
\hline & $\begin{array}{l}\text { Complex heart defect (hypoplastic left } \\
\text { heart, atrial septal defect, and aortic } \\
\text { atresia); right kidney fused to lower pole } \\
\text { of left kidney }\end{array}$ & Not applicable & $\begin{array}{l}\text { De novo nonsense mutation in the MAP4K4 } \\
\text { gene, which has recently been shown to be } \\
\text { important for endothelial cell migration and } \\
\text { vascular angiogenesis; this gene has not } \\
\text { previously been associated with human disease }\end{array}$ & $\begin{array}{l}\text { c. } 3568 C>T \\
\text { p. Gln1190* } \\
\text { de novo nonsense } \\
\text { novel mutation; VUS }\end{array}$ & $\begin{array}{l}\text { Uncertain- } \\
\text { other (novel } \\
\text { gene) }\end{array}$ \\
\hline & Nonimmune hydrops & Not applicable & $\begin{array}{l}\text { No identifiable etiology using exome } \\
\text { sequencing }\end{array}$ & & Negative \\
\hline & Shortened long bones & Not applicable & $\begin{array}{l}\text { No identifiable etiology using exome } \\
\text { sequencing }\end{array}$ & & Negative \\
\hline & Renal agenesis & Not applicable & $\begin{array}{l}\text { No identifiable etiology using exome } \\
\text { sequencing }\end{array}$ & & Negative \\
\hline & $\begin{array}{l}\text { Heterotaxy with a complex heart defect } \\
\text { and omphalocele }\end{array}$ & Not applicable & $\begin{array}{l}\text { No identifiable etiology using exome } \\
\text { sequencing }\end{array}$ & & Negative \\
\hline & $\begin{array}{l}\text { Multiple pregnancies with genitourinary } \\
\text { abnormalities }\end{array}$ & Not applicable & $\begin{array}{l}\text { No identifiable etiology using exome } \\
\text { sequencing }\end{array}$ & & Negative \\
\hline
\end{tabular}

AR Het, autosomal recessive-heterozygous; VUS, variant of uncertain significance. 
Table 4 Details of variants identified in positive and possible cases
Case Gene
Diagnosis
Alteration
Inheritance Classification Origin
Previously reported
Notes

ID

1 COL1A1 Osteogenesis c. $1875+1 \mathrm{G}>\mathrm{A}$

splice site variant

2 KCTD1 Scalp-ear-nipple $\quad$ c. $86 \mathrm{~A}>\mathrm{G}$

syndrome p. Asn29Ser

Likely

De novo Yes in a father and daughter with

osteogenesis imperfecta, type $1^{40}$ primordial dwarfism Thr1398Met

c. $5143 \mathrm{~A}>\mathrm{G}$

p. Asn1715Asp

4 MUSK

Fetal akinesia

pathogenir

De novo No

Rare variant at a canonical RNA splice donor site

Rare missense variant highly conserved in the BTB domain; several nearby misense changes with the same BTB domain have previously been associated with scalp - ear - nipple syndrome; anomalies of the kidneys have been reported and the neonatal demise showed several areas of skin sloughing and hypoplastic nails consistent with the previously described phenotype ${ }^{41}$ Both rare, highly conserved missense variants; other studies also report missense variants in this gene; RTTN is important in maintaining ciliary structure ${ }^{42-44}$

sequence

5 DYNC2H1 Short rib polydactyly $\quad$ c. 8012 T>C syndrome

p. Met2671Thr

AR

VUS

Inherited Yes, in an individual with short rib thoracic dysplasia type 3 with or without polydactyly.

$\begin{array}{ll}\text { c. } 10594 \mathrm{C}>\mathrm{T} & \text { Likely } \\ \text { p. Arg3532Ter } & \text { pathogenic }\end{array}$

\begin{tabular}{|c|c|c|c|c|c|c|}
\hline 6 & PIEZO1 & $\begin{array}{l}\text { Nonimmune } \\
\text { hydrops }\end{array}$ & $\begin{array}{l}\text { c. } 7129+1 G>C \\
\text { splice site variant }\end{array}$ & $A R$ & VUS; & Inherited \\
\hline & & & $\begin{array}{l}\text { c. } 307 C>T \\
\text { p. Arg103Ter }\end{array}$ & & $\begin{array}{l}\text { Likely } \\
\text { pathogenic }\end{array}$ & \\
\hline 7 & TMEM67 & $\begin{array}{l}\text { Meckel - Gruber } \\
\text { syndrome }\end{array}$ & $\begin{array}{l}\text { c. } 579 \text { 580delAG } \\
\text { p.Gly195fs }\end{array}$ & $A R$ & $\begin{array}{l}\text { Known } \\
\text { pathogenic }\end{array}$ & Inherited \\
\hline & & & $\begin{array}{l}\text { c. } 622 \mathrm{~A}>\mathrm{T} \\
\text { 0. Arg208Ter }\end{array}$ & & $\begin{array}{l}\text { Known } \\
\text { pathogenic }\end{array}$ & \\
\hline
\end{tabular}

Yes, previously reported as homozygous in a Predicted to result in premature truncation of the fetus with Meckel - Gruber syndrome and in protein two individuals with Joubert syndrome with limited clinical information ${ }^{47,48}$

Yes, previously reported in several fetuses with Meckel - Gruber syndrome ${ }^{49,50}$

Nonsense mutation; predicted to result in premature truncation of the protein

Rare missense variant in the AAA kinase domain previously reported in an individual with a similar phenotype; however, without further clinical or functional information, it is a VUS

Rare nonsense mutation predicted to result in therefore likely pathogenic

0. Arg208Ter

pathogenic p. Ile575Thr

pathogenic

c. $2408 \mathrm{~A}>\mathrm{G}$ VUS No

p. Tyr803Cys

Dutch founder mutation in 14 fetuses with fetal akinesia deformation sequence; lle575Thr disrupts multiple functions of the encoded protein ${ }^{45,46}$

Novel missense variant that alters a well-conserved amino acid from a tyrosine to a cysteine within the tyrosine kinase domain of the protein premature protein truncation; other truncating variants in this gene have been shown to cause disease and it is

Refs. 40-50 are in the Supplementary References online.

$\vec{\sim} \quad A D$, autosomal dominant; AR, autosomal recessive; BTB, bric-a-brac, tram track, and broad complex; VUS, variant of uncertain significance. 
human disease, the clinical significance of this variant is uncertain.

In two other cases, a single mutation in a gene associated with autosomal recessive inheritance of a phenotype consistent with the fetal presentation was identified. Incomplete sequencing coverage and the possibility of undetected deletions or duplications beyond that which could be detected by microarray precluded the exclusion of a second mutation.

We found only one medically actionable finding in a parent (familial hypercholesterolemia $(L D L R)$ ), which was confirmed with Sanger sequencing. The parent in this case was already being treated for high cholesterol and had a strong family history of hypercholesterolemia. The participant was encouraged to share the information with family members in the posttest counseling session. None of the couples had significant carrier results to report. Two couples chose to have fetal results placed in the medical records and they plan to have prenatal diagnosis in a future pregnancy if the same anomalies are noted.

The mothers' self-reporting of knowledge and attitudes revealed a median perceived likelihood of 5.2 on a 10-point Likert scale (range: 2-7) that exome sequencing would provide a result for the abnormalities identified in a couple's fetus. The median genomic knowledge before sequencing was high (92.0; range: 76.0-100.0). The study was not powered to detect a difference in genetics knowledge base assessment by socioeconomic background, but there was a statistically significant finding that women in the highest socioeconomic group (those with a family income of more than $\$ 90,000$ annually) had higher presequencing genomic knowledge (median: 95.0; 95\% CI: 91.6-98.4) than their lower-income counterparts (those with a family income of less than $\$ 90,000$ annually; median: 88.0; 95\% CI: 85.0-92.6; $P<0.001$ ). Seventy-five percent of the women who scored above the mean were in the highest income bracket. In the post-results surveys and interviews, all of the women expressed an understanding of their exome sequencing results and felt that they had made a good decision in having exome sequencing performed. In a qualitative interview, the parent who received the incidental finding expressed the view that having exome sequencing was beneficial to his long-term health.

\section{DISCUSSION}

Our series of noncontinuing anomalous pregnancies shows that the diagnostic utility of exome sequencing after normal standard genetic testing yields a definitive or possible explanation in up to $47 \%(7 / 15)$ of cases where a fetal genetic diagnosis was highly suspected. This is on the higher end of prenatal yields reported by other authors of similarly small series, which range from 10 to $57 \%$, and confirms that exome sequencing increases the diagnostic yield prenatally in a select group of anomalous fetuses who fail to receive a diagnosis with standard genetic testing. ${ }^{8-10,24}$ The criteria for study inclusion, sample sizes, and diagnostic yields of other published studies are as follows: fetal demise or termination of pregnancy with multiple congenital anomalies with normal karyotype results using trios ( $n=7$; diagnostic yield: $57 \%) ;{ }^{10}$ increased nuchal translucency $(>3.5 \mathrm{~mm})$ and/or another abnormality with normal karyotype results $(n=24$; diagnostic yield: $21 \%) ;{ }^{8}$ and diverse structural abnormalities on ultrasound using trios $(n=30$; diagnostic yield: $10 \%){ }^{24}$ It is important to note that the diagnostic yield of any test depends on the previous probability of detectable conditions within that cohort, so it is likely that our apparently higher yield and that of Alamillo et al. ${ }^{10}$ reflect the inclusion of fetuses with a higher likelihood of a genetic etiology given that both studies only included fetuses in noncontinuing pregnancies with multiple congenital anomalies. In addition, both our study and that of Alamillo et al. used trios consistently, which improves diagnostic rates. The other studies with lower yields included fetuses with a single structural abnormality. The yield in a larger sample with broader inclusion criteria may be lower. In addition to the selection of a cohort with a high likelihood of genetic etiologies, the interpretation of findings also influences diagnostic yield. Our approach was consistent with guidelines by Richards et al. and used pre-established multidisciplinary variant analysis committees put in place for the NCGENES project; thus, we do not feel our diagnostic yield was overinflated. Our study performed both karyotyping and microarrays on all included fetuses, whereas other studies did not consistently perform microarrays. Given that exome sequencing cannot detect larger copy-number variants, we felt it was important that chromosomal microarrays be done before exome sequencing. Our findings suggest that exome sequencing improves the accuracy of prenatal diagnosis in a select cohort of fetuses with multiple congenital abnormalities because exome sequencing has an increased diagnostic capability when karyotyping and microarrays are normal.

The strengths of our study include the use of trios, which enhances diagnostic yield and was not consistently used by other studies, ${ }^{8,25}$ the development of fetal-specific gene lists to optimize turnaround time, the development and use of triospecific bioinformatics pipelines, and the use of a multidisciplinary genetics team to evaluate the classification of all reported results with respect to the pathogenicity of the variants and (for the diagnostic results) the likelihood that those variants explained the phenotype. ${ }^{8,25}$ Our study also found that exome sequencing was useful in cases where a clinically available phenotype-driven panel did not provide an answer. We identified variants in genes that were not on the specific prenatal panel for the phenotype being tested, either because the gene had not been described at the time the panel was validated or because the phenotype was so heterogeneous that a complete panel could not be made (e.g., for hydrops). Since we included only cases of noncontinuing pregnancies, the postnatal exam of the fetus by a geneticist with autopsy was available in some cases to assist in refining the phenotype. This allowed us to specifically target genes associated with a particular phenotype and added confidence when pathogenic variants were identified. Our study, along with previous studies, provides pilot data indicating that exome sequencing can improve prenatal diagnosis. 
Given the important counseling issues inherent in exome sequencing, we also explored the important and critical issue regarding how mothers perceive and understand exome sequencing. Efforts to understand the psychosocial and behavioral impact of integrating genomic technologies into adult and pediatric practice are ongoing. ${ }^{26-28}$ To date, little empirical work has been done to understand the unique challenges of applying exome or genome sequencing to the prenatal context. The experience of prenatal diagnosticians and patients regarding responses to variants of uncertain significance and the incidental identification of maternal pathology after prenatal chromosomal microarray has been studied, and raises a range of similar issues. ${ }^{29,30}$ These include complexities of trade-offs between better diagnostic ability than standard karyotyping ${ }^{31}$ and a greater risk of producing results with uncertain clinical significance. While prenatal diagnosticians have incorporated pre- and posttest counseling into their practices to explain nuanced results, the issues are magnified by the use of exome sequencing in this population, given the higher incidence of uncertain variants in a sequencing context.

We found that women with a lower family income scored significantly lower on the genetics literacy assessment compared with women with a higher family income. We also found that women had high hopes and expectations (Likert scale: 5.2) that exome sequencing would provide a result despite pretest counseling by a genetic counselor during which it was explained that exome sequencing has previously been shown to yield a result approximately $30 \%$ of the time. However, when using a Likert scale, participants may choose the neutral option because picking a neutral option allows people to avoid the cognitive effort needed to choose between their positive and negative feelings on an issue. ${ }^{21}$ Attitudes towards prenatal screening and diagnosis are influenced by ethnicity, socioeconomic status, cultural and religious beliefs, acceptability of termination of pregnancy, and experiences with disability. Further research on this critical topic is needed to ensure that patients' needs are being met as new technologies inevitably become implemented in clinical practice. $^{32-34}$

Our study also demonstrates that exome sequencing in this context can extend the understanding of known and novel diseases that disrupt fetal development. The finding of a likely pathogenic variant in KCTD1 expands the phenotype of a known Mendelian disorder (scalp - ear - nipple syndrome) to the fetal period. The discovery of a de novo truncating mutation in MAP4K4 in a fetus with a complex heart defect makes this a novel candidate gene for a human developmental disorder, given its critical role in embryonic development of the heart in mouse models. ${ }^{23,35,36}$ Further supporting the possibility of this gene as causative of the described phenotype is its de novo status. Further in vivo studies are planned using a zebrafish model to explore this intriguing finding.

The limitations of our study include its relatively small sample size and the selection of cases with a high a priori likelihood of having a genetic etiology. As the cost decreases, exome sequencing may become more cost-effective than pursuing multigene panels, although analytic considerations, such as depth of coverage and coverage across exons, may be optimal with molecular panels. Our study was not powered to identify statistical differences in outcomes related to maternal expectations and understanding; this is an area that needs further exploration in larger clinical studies of prenatal exome sequencing, especially given that trends from this study suggest that knowledge scores are related to socioeconomic status.

While exome sequencing is a promising diagnostic technology in the prenatal, childhood, and adult settings, there remain important limitations and ethical issues surrounding its use, including the provision of adequate counseling and informed consent. False negatives should be expected with exome sequencing given that most platforms cover only 85 to $90 \%$ of exons. Turnaround time has been cited as an issue when exome sequencing is applied prenatally, but the use of phenotype-specific gene lists and trio analysis, as in the current study, has substantially decreased the turnaround time. ${ }^{37}$ Certainly, before exome sequencing is routinely implemented prenatally, the turnaround time needs to be optimized so that reproductive decisions can be made in a timely manner. There are also ethical issues related to triosequencing, including disclosure of nonpaternity, consanguinity, and medically actionable findings in parents. In addition, if exome sequencing is applied in ongoing pregnancies, the additional ethical issue of being able to report a predisposition to adult onset disorders from fetal information arises. These issues will require ongoing ethical consideration, as well as access to comprehensive genetic counseling by a certified genetic counselor with prenatal experience.

The results of the current study show that exome sequencing provides information to families, expands clinical phenotypes to the fetal period, and will likely enhance our knowledge of genes critical to fetal development. Neither the American College of Medical Genetics and Genomics nor the American College of Obstetrics and Gynecology recommends that exome sequencing be used routinely. ${ }^{20,38}$ Questions about the most cost-effective and efficient way of identifying pathogenic variants in fetuses that do not receive a result with chromosomal microarray should be addressed in larger clinical trials. Given the importance of responsibly applying new technologies to the broadest population possible, including traditionally underserved patients, decision aids in conjunction with genetic counseling sessions should be developed and studied to determine whether these interventions improve the understanding of the types of results exome sequencing may provide. Further studies on both diagnostic utility and maternal expectations and understanding of prenatal exome sequencing are crucial before this technology becomes routinely incorporated into prenatal care.

\section{SUPPLEMENTARY MATERIAL}

Supplementary material is linked to the online version of the paper at http://www.nature.com/gim 


\section{ACKNOWLEDGMENTS}

We thank Kathleen Kaiser-Rogers and the UNC-Chapel Hill Cytogenetics Laboratory, Elysia Davis, Karen Dorman, Erin Eaton, Ginger Hocutt, Manyu Li, Amber Ivins, Patricia Basta and the Biospecimen Processing Facility, and Diane Vargo. The authors received a National Institutes of Health Clinical and Translational Science Award from UNC at Chapel Hill (TTR11403) and a Building Interdisciplinary Research in Women's Health award from the National Institute of Child Health and Human Development (2K12HD00144116), as well as funding from the National Human Genome Research Institute (HG006487; presented at the American Society of Human Genetics; Vancouver, British Columbia, 17-22 October 2016). The NHGRI award is to Jim Evans and Jonathan Berg.

\section{DISCLOSURE}

The authors declare no conflict of interest.

\section{REFERENCES}

1. Osterman MJ, Kochanek KD, MacDorman MF, Strobino DM, Guyer B. Annual summary of vital statistics: 2012-2013. Pediatrics 2015;135: $1115-1125$.

2. Shaffer LG, Rosenfeld JA, Dabell MP, et al. Detection rates of clinically significant genomic alterations by microarray analysis for specific anomalies detected by ultrasound. Prenat Diagn 2012;32:986-995.

3. Van den Veyver IB, Eng CM. Genome-wide sequencing for prenatal detection of fetal single-gene disorders. Cold Spring Harb Perspect Med 2015;5:a023077.

4. Yang Y, Muzny DM, Reid JG, et al. Clinical whole-exome sequencing for the diagnosis of mendelian disorders. N Engl J Med 2013;369: 1502-1511.

5. Cukier HN, Dueker ND, Slifer SH, et al. Exome sequencing of extended families with autism reveals genes shared across neurodevelopmental and neuropsychiatric disorders. Mol Autism 2014;5:1

6. Wang $H$, Sun $Y$, Wu W, Wei X, Lan Z, Xie J. A novel missense mutation of FGFR3 in a Chinese female and her fetus with hypochondroplasia by next-generation sequencing. Clin Chim Acta 2013;423:62-65.

7. Filges I, Nosova E, Bruder E, et al. Exome sequencing identifies mutations in KIF14 as a novel cause of an autosomal recessive lethal fetal ciliopathy phenotype. Clin Genet 2014;86:220-228.

8. Drury $\mathrm{S}$, Williams $\mathrm{H}$, Trump $\mathrm{N}$, et al. Exome sequencing for prenatal diagnosis of fetuses with sonographic abnormalities. Prenat Diagn 2015;35:1010-1017.

9. Hillman SC, Willams D, Carss KJ, McMullan DJ, Hurles ME, Kilby MD. Prenatal exome sequencing for fetuses with structural abnormalities: the next step. Ultrasound Obstet Gynecol 2015;45:4-9.

10. Alamillo $C L$, Powis $Z$, Farwell $K$, et al. Exome sequencing positively identified relevant alterations in more than half of cases with an indication of prenatal ultrasound anomalies. Prenat Diagn 2015;35:1073-1078.

11. Green RC, Berg JS, Grody WW, et al. ACMG recommendations for reporting of incidental findings in clinical exome and genome sequencing. Genet Med 2013;15:565-574.

12. Jung CH, Lee MJ, Kang YM, et al. 2013 ACC/AHA versus 2004 NECP ATP III guidelines in the assignment of statin treatment in a Korean population with subclinical coronary atherosclerosis. PloS ONE 2015;10:e0137478.

13. Renaissance Computing Institute Technologies for Genomic Medicine. CANVAS and AnnoBot, Solutions for Genomic Variant Annotation. http://www.renci.org/TR-14-04. Accessed 24 March 2014.

14. Cingolani $P$, Platts $A$, Wang le $L$, et al. A program for annotating and predicting the effects of single nucleotide polymorphisms, SnpEff: SNPs in the genome of Drosophila melanogaster strain w1118; iso-2; iso-3. Fly (Austin) 2012;6:80-92.

15. Walsh $\mathrm{R}$, Thomson KL, Ware JS, et al. Reassessment of Mendelian gene pathogenicity using 7,855 cardiomyopathy cases and 60,706 reference samples. Genet Med 2017;19:192-203.
16. Paila U, Chapman BA, Kirchner R, Quinlan AR. GEMINI: integrative exploration of genetic variation and genome annotations. PLoS Comput Biol 2013;9:e1003153.

17. Richards S, Aziz N, Bale S, et al. Standards and guidelines for the interpretation of sequence variants: a joint consensus recommendation of the American College of Medical Genetics and Genomics and the Association for Molecular Pathology. Genet Med 2015;17: 405-424.

18. Berg JS, Adams M, Nassar N, et al. An informatics approach to analyzing the incidentalome. Genet Med 2013;15:36-44.

19. Retterer K, Juusola J, Cho MT, et al. Clinical application of whole-exome sequencing across clinical indications. Genet Med 2016;18:696-704.

20. Vora NL RS, Ralston SJ, Dugoff L, Kuller JA. Microarrays and nextgeneration sequencing technology: the use of advanced genetic diagnostic tools in obstetrics and gynecology. Obstet Gynecol 2016;128: e262-e268.

21. Strande NT, Berg JS. Defining the clinical value of a genomic diagnosis in the era of next-generation sequencing. Annu Rev Genomics Hum Genet 2016;17:303-332.

22. Kalton GG, Roberts J, Holt, DD. The effects of offering a middle response option with opinion questions. J $R$ Stat Soc 1980;29:65-78.

23. Vitorino $P$, Yeung $S$, Crow $A$, et al. MAP4K4 regulates integrin-FERM binding to control endothelial cell motility. Nature 2015;519:425-430.

24. Carss KJ, Hillman SC, Parthiban V, et al. Exome sequencing improves genetic diagnosis of structural fetal abnormalities revealed by ultrasound. Hum Mol Genet 2014;23:3269-3277.

25. Pangalos C, Hagnefelt B, Lilakos K, Konialis C. First applications of a targeted exome sequencing approach in fetuses with ultrasound abnormalities reveals an important fraction of cases with associated gene defects. PeerJ. 2016;4:e1955.

26. Gray SW, Martins Y, Feuerman LZ, et al. Social and behavioral research in genomic sequencing: approaches from the Clinical Sequencing Exploratory Research Consortium Outcomes and Measures Working Group. Genet Med 2014;16:727-735.

27. Kaphingst KA, Ivanovich J, Biesecker BB, et al. Preferences for return of incidental findings from genome sequencing among women diagnosed with breast cancer at a young age. Clin Genet 2016;89:378-384.

28. Biesecker $B B$, Klein $W$, Lewis $K L$, et al. How do research participants perceive "uncertainty" in genome sequencing? Genet Med 2014;16: 977-980.

29. Reiff M, Bernhardt BA, Mulchandani $S$, et al. "What does it mean?": uncertainties in understanding results of chromosomal microarray testing. Genet Med 2012;14:250-258.

30. Bernhardt BA, Soucier D, Hanson K, Savage MS, Jackson L, Wapner RJ. Women's experiences receiving abnormal prenatal chromosomal microarray testing results. Genet Med 2013;15:139-145.

31. Wapner RJ, Martin CL, Levy $B$, et al. Chromosomal microarray versus karyotyping for prenatal diagnosis. N Engl J Med 2012;367: 2175-2184.

32. Case AP, Ramadhani TA, Canfield MA, Wicklund CA. Awareness and attitudes regarding prenatal testing among Texas women of childbearing age. J Genet Couns 2007;16:655-661.

33. Kuppermann M, Gates E, Washington AE. Racial-ethnic differences in prenatal diagnostic test use and outcomes: preferences, socioeconomics, or patient knowledge? Obstet Gynecol 1996;87:675-682.

34. Kuppermann M, Nakagawa S, Cohen SR, Dominguez-Pareto I, Shaffer $\mathrm{BL}$, Holloway SD. Attitudes toward prenatal testing and pregnancy termination among a diverse population of parents of children with intellectual disabilities. Prenat Diagn 2011;31:1251-1258.

35. Su YC, Treisman JE, Skolnik EY. The Drosophila Ste20-related kinase misshapen is required for embryonic dorsal closure and acts through a JNK MAPK module on an evolutionarily conserved signaling pathway. Genes Dev 1998;12:2371-2380.

36. Xue Y, Wang X, Li Z, Gotoh N, Chapman D, Skolnik EY. Mesodermal patterning defect in mice lacking the Ste20 NCK interacting kinase (NIK). Development 2001;128:1559-1572.

37. Saunders CJ, Miller NA, Soden SE, et al. Rapid whole-genome sequencing for genetic disease diagnosis in neonatal intensive care units. Sci Transl Med 2012;4:154ra135

38. ACMG Board of Directors Points to consider in the clinical application of genomic sequencing. Genet Med 2012;14:759-761. 